\title{
A Novel Method to Adjust Efficacy Estimates for Uptake of Other Active Treatments in Long-Term Clinical Trials
}

\author{
John Simes ${ }^{1 *}$, Merryn Voysey ${ }^{1}$, Rachel O'Connell $^{1}$, Paul Glasziou ${ }^{2}$, James D. Best ${ }^{3}$, Russell Scott ${ }^{4}$, \\ Christopher Pardy ${ }^{1}$, Karen Byth ${ }^{1}$, David R. Sullivan ${ }^{5}$, Christian Ehnholm ${ }^{6}$, Anthony Keech ${ }^{1}$, for the FIELD \\ Study Investigators
}

1 National Health and Medical Research Council Clinical Trials Centre, University of Sydney, Sydney, Australia, 2 Centre for Evidence-Based Medicine, University of Oxford, Oxford, United Kingdom, 3 Faculty of Medicine, University of Melbourne, Melbourne, Australia, 4 Lipid and Diabetes Research Group, Christchurch Hospital, Christchurch, New Zealand, 5 Department of Clinical Biochemistry, Royal Prince Alfred Hospital, Sydney, Australia, 6 Biomedicum Helsinki, National Public Health Institutes, Helsinki, Finland

\begin{abstract}
Background: When rates of uptake of other drugs differ between treatment arms in long-term trials, the true benefit or harm of the treatment may be underestimated. Methods to allow for such contamination have often been limited by failing to preserve the randomization comparisons. In the Fenofibrate Intervention and Event Lowering in Diabetes (FIELD) study, patients were randomized to fenofibrate or placebo, but during the trial many started additional drugs, particularly statins, more so in the placebo group. The effects of fenofibrate estimated by intention-to-treat were likely to have been attenuated. We aimed to quantify this effect and to develop a method for use in other long-term trials.

Methodology/Principal Findings: We applied efficacies of statins and other cardiovascular drugs from meta-analyses of randomized trials to adjust the effect of fenofibrate in a penalized Cox model. We assumed that future cardiovascular disease events were reduced by an average of $24 \%$ by statins, and $20 \%$ by a first other major cardiovascular drug. We applied these estimates to each patient who took these drugs for the period they were on them. We also adjusted the analysis by the rate of discontinuing fenofibrate. Among 4,900 placebo patients, average statin use was $16 \%$ over five years. Among 4,895 assigned fenofibrate, statin use was $8 \%$ and nonuse of fenofibrate was $10 \%$. In placebo patients, use of cardiovascular drugs was $1 \%$ to $3 \%$ higher. Before adjustment, fenofibrate was associated with an $11 \%$ reduction in coronary events (coronary heart disease death or myocardial infarction) $(P=0.16)$ and an $11 \%$ reduction in cardiovascular disease events $(P=0.04)$. After adjustment, the effects of fenofibrate on coronary events and cardiovascular disease events were $16 \%(P=0.06)$ and $15 \%(P=0.008)$, respectively.

Conclusions/Significance: This novel application of a penalized Cox model for adjustment of a trial estimate of treatment efficacy incorporates evidence-based estimates for other therapies, preserves comparisons between the randomized groups, and is applicable to other long-term trials. In the FIELD study example, the effects of fenofibrate on the risks of coronary heart disease and cardiovascular disease events were underestimated by up to one-third in the original analysis.
\end{abstract}

Trial Registration: Controlled-Trials.com ISRCTN64783481

Citation: Simes J, Voysey M, O'Connell R, Glasziou P, Best JD, et al. (2010) A Novel Method to Adjust Efficacy Estimates for Uptake of Other Active Treatments in Long-Term Clinical Trials. PLoS ONE 5(1): e8580. doi:10.1371/journal.pone.0008580

Editor: Benjamin Djulbegovic, University of South Florida, United States of America

Received September 7, 2009; Accepted November 26, 2009; Published January 8, 2010

Copyright: (c) 2010 Simes et al. This is an open-access article distributed under the terms of the Creative Commons Attribution License, which permits unrestricted use, distribution, and reproduction in any medium, provided the original author and source are credited.

Funding: Fenofibrate Intervention and Event Lowering in Diabetes was supported by grants from Laboratoires Fournier SA, Dijon, France (now part of Solvay Pharmaceuticals), and the National Health and Medical Research Council, Australia. The funders had no role in study design, data collection and analysis, decision to publish, or preparation of the manuscript.

Competing Interests: Research grant awarded to University of Sydney from Laboratoire Fournier, and honoraria for invited addresses from Solvay Pharmaceuticals.

*E-mail: john@ctc.usyd.edu.au

\section{Introduction}

A common problem in longer-term clinical trials comparing chronic treatments is that patients may start using additional active therapies during the course of the trial, which may confound the evaluation of the trial's target treatments $[1,2]$. This is particularly the case when the uptake of such therapies differs between the treatment arms, potentially resulting in an underestimation of the direct benefit of the target treatment. Additional therapies may be given to patients as a result of new evidence emerging from other ongoing trials or because of changes in patient and clinician choice over time, and also to those responding poorly to initial treatments, as in some cancer trials [3]. Patients may discontinue trial treatments for similar reasons.

Conventional methods in clinical trials either use intention-totreat analysis only or adjust for changes in treatment after randomization (such as in per-protocol analyses). The former may underestimate the true biological effect of treatment because of noncompliance, and the latter may be confounded by the differences between those patients who do and those who do not 
adhere to their randomized treatments or between those who do and those who do not take up other therapies. These latter analyses are prone to selection bias, in that they do not maintain the randomized structure of the comparisons $[1,4,5]$.

This specific problem arose in the analysis of the 5 -year Fenofibrate Intervention and Event Lowering in Diabetes (FIELD) trial - a large-scale trial of the lipid-modifying effects of fenofibrate compared with placebo in patients with type 2 diabetes mellitus [6]. The study design was pragmatic in evaluating the effect of fenofibrate on a background of usual medical care [6,7]. This meant that in the light of new clinical circumstances or the emergence of new evidence, additional cardiovascular medicines, including statins and other lipid modifying treatments, could be commenced during the course of the trial. Methods have been proposed to account for noncompliance with randomized treatment $[1,8]$ (including instrumental variable analysis [9-11]), but these methods do not deal with the situation we encountered in FIELD of a large imbalance between the treatment groups in the proportion of patients who commenced active nonstudy medications. In the standard intention-to-treat analysis, unbalanced uptake of nonstudy treatment can attenuate the estimated effect of the study drug. Measuring the influence of the uptake of nonstudy medications requires estimates of the effect of these medications from sources external to the trial in question [1], as any estimates derived from within the trial are subject to selection bias. In this analysis, using FIELD as our example, we report a novel method for incorporating external evidence-based estimates to correct for this.

Adjustment for any dilution of the treatment effect caused by discontinuation of the randomized study drug by some patients was also examined by using a randomization-based efficacy estimator to adjust for nonadherence to study treatment [1].

\section{Methods}

\section{FIELD Trial Design}

FIELD was a randomized double-blind placebo-controlled trial in 9795 middle-aged to elderly people with type 2 diabetes mellitus $[6,7,12]$ After a 16 -week run-in period, patients were randomized to micronised fenofibrate (200 mg daily) or matching placebo and followed up through regular clinic visits in addition to usual care from their treating doctors for a planned median duration of 5 years.

During the course of the trial and before any unblinding of results, the trial's progress was monitored for rates of commencement of open-label lipid treatment, adherence to study treatment, and cardiovascular events (for both treatment groups combined). In the light of emerging evidence of the effectiveness of statin therapy, the increased uptake of statin treatment in the trial, and a lower than expected pooled event rate, the primary outcome, coronary heart disease (CHD) death, was revised in 2002 to CHD events (CHD death or myocardial infarction) [6]. The revised trial design was powered to detect a 22\% reduction in CHD events (based on intention-to-treat analysis). This corresponded to a $27 \%$ reduction among those on treatment (based on a per-protocol analysis).

\section{Patient Population and Treatments}

Patients with diabetes, with or without pre-existing cardiovascular disease or lipid abnormalities, were eligible, provided total blood cholesterol level at screening was 3.0 to $6.5 \mathrm{mmol} / \mathrm{L}$, and either the triglyceride level was between 1.0 and $5.0 \mathrm{mmol} / \mathrm{L}$ or the total cholesterol/high-density lipoprotein cholesterol ratio was 4.0 or higher. Lipid values at screening were provided to the patients' doctors before randomization: all patients for whom any cholesterol-lowering treatment (including statins) was indicated at the start of the trial were ineligible. However, these (and other) medications could be commenced after randomization if the usual doctor considered it appropriate (for example, because of changed clinical circumstances) [12].

Cardiovascular medications were recorded at each follow-up visit (at least 6 monthly), as was adherence to study treatment.

\section{Cardiovascular Outcomes and Subgroups}

The primary study endpoint was the first occurrence of CHD death or nonfatal myocardial infarction. Secondary outcomes included major cardiovascular events (CHD events, total stroke and other cardiovascular death combined), total cardiovascular events (major cardiovascular events plus coronary and carotid revascularization), GHD death, total cardiovascular deaths, stroke, and coronary and peripheral revascularization procedures.

The adjusted effect of fenofibrate on total cardiovascular events was examined within the main subgroups - men vs women, those aged $<65$ years vs those aged $\geq 65$ years, and the presence vs absence of prior cardiovascular disease - to see whether differential uptake of other medicines by subgroup affected these comparisons.

\section{Estimates of Treatment Effect of Statins and Other Cardiovascular Drugs}

The effectiveness of various medications in preventing cardiovascular events has been well established in several randomized controlled trials (Table S1) [13-20], with estimates of reductions in events in various settings ranging from $16 \%$ to $63 \%$. The effect of combinations of drugs has, for the most part, been observed to be multiplicative on the basis of a similar relative risk reduction in randomized trials in the presence or absence of other drugs [14,21].

In this analysis, evidence for the effectiveness of statins and other cardiovascular medicines was based on published systematic reviews of randomized trials of these therapies for diabetes populations and, in the absence of heterogeneity of treatment effects, for broader populations at risk of cardiovascular disease. Effects of the following medicines or classes of drugs were used in the adjusted analyses: statin drugs (simvastin, atorvastatin, pravastastin, any other statin); angiotensin-converting enzyme (ACE) inhibitors or angiotensin II receptor blockers; beta blockers; calcium-channel antagonists; diuretics; antiplatelet drugs (aspirin or other).

The estimate of the effect of statin use on subsequent cardiovascular events was based on the estimate of the Cholesterol Treatment Trialists' Collaboration's (CTTC) systematic overview of 14 large-scale randomized trials of statin therapy: a $21 \%$ reduction in cardiovascular events per $\mathrm{mmol} / \mathrm{L}$ reduction in lowdensity lipoprotein (LDL) cholesterol [22]. Subgroup meta-analysis showed no heterogeneity of the statin treatment effect between those with and those without diabetes [23].

We estimated the absolute reduction in LDL cholesterol by statin therapy by applying the average percentage reduction in LDL cholesterol, estimated from a meta-analysis of 164 short-term randomized trials [24], to the LDL cholesterol levels of each treatment group (fenofibrate or placebo) in our cohort before they started any statin therapy. This average percentage reduction in LDL cholesterol was weighted according to the different statin drugs taken and the average dose of each used within each treatment group. The assumed event reduction (for each type of event) was then determined as the relative reduction in events per $\mathrm{mmol} / \mathrm{L}$ reduction in LDL cholesterol multiplied by the average absolute reduction in LDL cholesterol.

For other cardiovascular drugs (ACE inhibitors, beta blockers, calcium-channel blockers, diuretics and antiplatelet drugs), a more simple, yet conservative, approach was taken. There was an assumed $20 \%$ reduction in the risk of any subsequent cardiovas- 
cular event due to the first nonstudy drug taken and a $15 \%$ reduction for each additional drug.

\section{Statistical Methods}

All patients were included in the randomized comparisons, and analyses were by intention-to-treat. As specified in the published study protocol, the unadjusted primary analyses for cardiovascular events used standard log-rank methods without adjustment for covariates [25], and Cox proportional-hazards modelling was used to compute hazard ratios (HRs) and their 95\% confidence intervals [26,27].

Adjustment for the use of other cardiovascular medications used a penalized Cox model [27], for which the general formula for the hazard function at time $t$ for patient $i$ is:

$$
\lambda_{\mathrm{i}}(t)=\lambda_{0}(t) e^{\mathrm{Xi} \beta+\mathrm{Zi}(\mathrm{t}) \omega}
$$

where $\lambda_{0}(t)$ is the baseline hazard function, $X_{i}$ is the covariate indicator for treatment group $(=1$ for fenofibrate and 0 for placebo) and $\mathrm{Z}_{\mathrm{i}}(\mathrm{t})$ is the covariate vector indicating usage of cardiovascular disease medicines at time $t$ for patient $i$. In this model $\beta$ is the parameter for the treatment effect of fenofibrate (unconstrained coefficient), while $\boldsymbol{\omega}$ is a vector of the assumed effects of other cardiovascular disease medicines (constrained coefficients). The HR from this model for the adjusted fenofibrate effect is estimated as $\exp (\hat{\beta})$.

When we adjusted for the effect of statins only, the constrained coefficient $\boldsymbol{\omega}$ (offset) in this formula was set to the log of the HR for the effect of statin therapy within the treatment group. For example, the evidence-based effect of statins on total cardiovascular events was estimated to be $25 \%$ in those on placebo, and thus for a placebo patient, $\boldsymbol{\omega}=\log (0.75)$. In the case of adjustment for other cardiovascular medications, the value of the offset was calculated on the basis of the number and type of medicines taken by each patient at any time $(20 \%$ reduction in risk from the first additional drug taken and a $15 \%$ reduction for each additional drug). The

Table 1. Use of the study drug and other medication (average \% over 5 years) by treatment group and major subgroup in the FIELD study $(n=9795)$.

\begin{tabular}{|c|c|c|c|c|c|}
\hline \multirow[b]{2}{*}{ Subgroup } & \multirow[b]{2}{*}{$\%$} & \multirow{2}{*}{$\begin{array}{l}\text { Discontinuedstudy drug } \\
\text { Placebo group }\end{array}$} & \multirow{2}{*}{$\begin{array}{l}\text { Discontinued study drug } \\
\text { Fenofibrate group }\end{array}$} & \multirow{2}{*}{$\begin{array}{l}\text { Started other lipid- } \\
\text { lowering treatment* }\end{array}$} & \multirow{2}{*}{$\begin{array}{l}\text { Started other lipid- } \\
\text { lowering treatment* } \\
\text { Fenofibrate group }\end{array}$} \\
\hline & & & & & \\
\hline \multicolumn{6}{|l|}{ Sex } \\
\hline Men & 63 & 9 & 10 & 17 & 9 \\
\hline Women & 37 & 10 & 11 & 18 & 7 \\
\hline \multicolumn{6}{|l|}{ Age (years) } \\
\hline$<65$ & 60 & 9 & 9 & 17 & 8 \\
\hline$\geq 65$ & 40 & 10 & 12 & 18 & 9 \\
\hline \multicolumn{6}{|l|}{ Previous CVD } \\
\hline Yes & 22 & 11 & 14 & 23 & 14 \\
\hline No & 78 & 9 & 9 & 16 & 7 \\
\hline \multicolumn{6}{|l|}{ Hypertension } \\
\hline Yes & 84 & 9 & 10 & 17 & 9 \\
\hline No & 16 & 10 & 10 & 16 & 7 \\
\hline \multicolumn{6}{|c|}{ Waist measurement } \\
\hline Hight & 68 & 10 & 10 & 17 & 9 \\
\hline Low & 32 & 9 & 11 & 17 & 7 \\
\hline \multicolumn{6}{|l|}{ Dyslipidemia } \\
\hline Yes: & 38 & 10 & 12 & 21 & 12 \\
\hline No & 62 & 9 & 10 & 15 & 6 \\
\hline \multicolumn{6}{|c|}{ HDL cholesterol (mmol/L) } \\
\hline High & 41 & 9 & 9 & 15 & 6 \\
\hline Low§ & 59 & 10 & 11 & 19 & 10 \\
\hline \multicolumn{6}{|c|}{ LDL cholesterol (mmol/L) } \\
\hline$<3.0$ & 45 & 9 & 11 & 11 & 6 \\
\hline $3.0-3.5$ & 29 & 9 & 10 & 17 & 9 \\
\hline$>3.5 \mathrm{mmol} / \mathrm{L}$ & 26 & 11 & 11 & 28 & 11 \\
\hline All patients & 100 & 10 & 10 & 17 & 8 \\
\hline
\end{tabular}

*Based on patients who took statins, resins, fibrates or other lipid-modifying drugs for at least 3 months.

Men: $\geq 102 \mathrm{~cm}$; women: $\geq 88 \mathrm{~cm}$.

tow HDL cholesterol plus high triglyceride ( $\geq 1.7 \mathrm{mmol} / \mathrm{L})$.

$\S<1.03 \mathrm{mmol} / \mathrm{L}$ for men, $<1.29 \mathrm{mmol} / \mathrm{L}$ for women.

FIELD = Fenofibrate Intervention and Event Lowering in Diabetes; CVD = cardiovascular disease; $\mathrm{HDL}=$ high-density lipoprotein; $L D L=$ low-density lipoprotein.

doi:10.1371/journal.pone.0008580.t001 


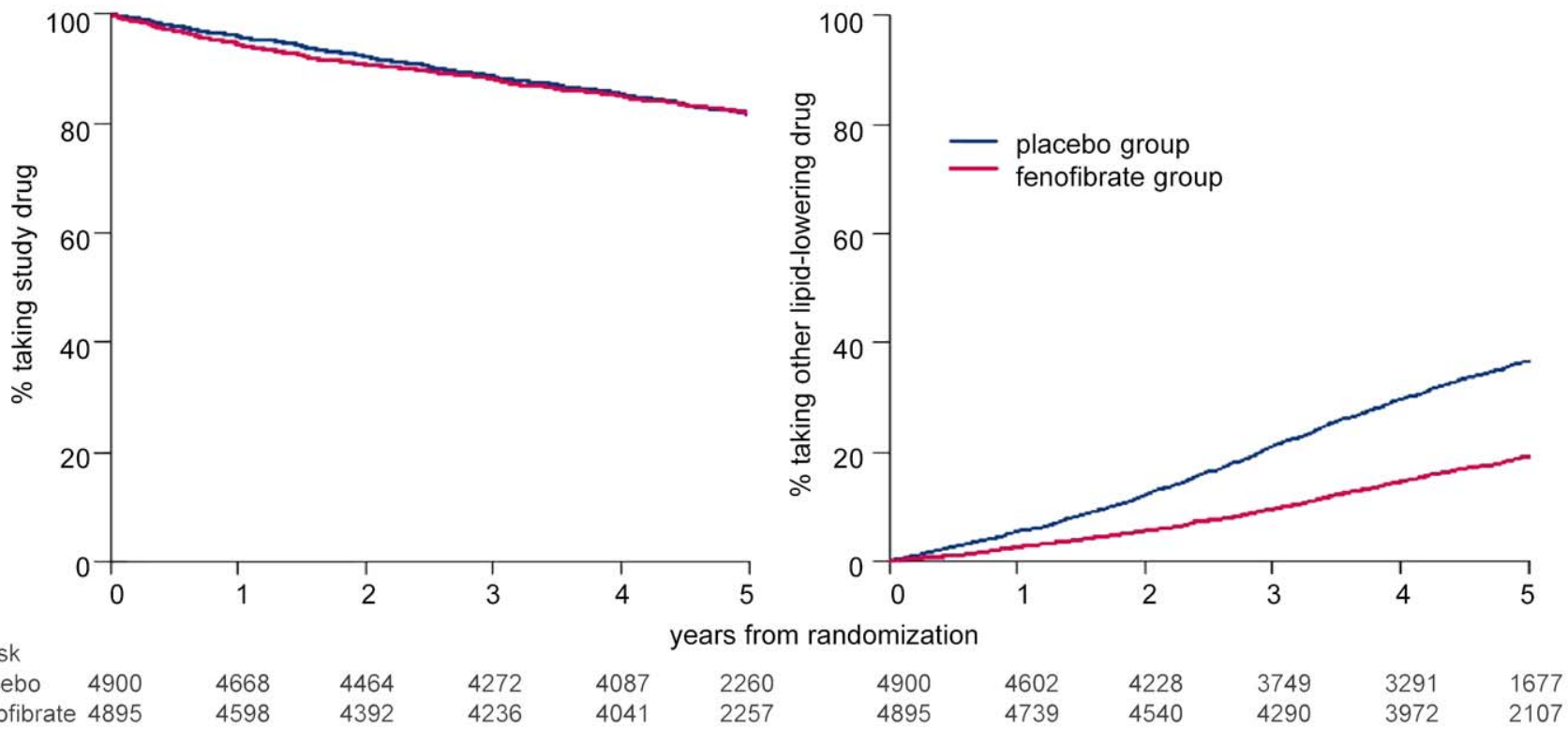

Figure 1. Time to discontinuing study medication or to starting other lipid-lowering treatment, by randomized group. doi:10.1371/journal.pone.0008580.g001

value of the offset in these cases was the addition of $\log (\mathrm{HR}$ for statins), if the patient was treated with statins, plus $\log (0.8)$ for the first additional drug plus $\log (0.85)$ for each subsequent drug. This value changed as the patient's prescriptions changed. For example, a patient taking a statin, an ACE inhibitor and a diuretic would have an offset value of $-0.673(\log (0.75 \times 0.8 \times 0.85))$ only for the period he or she was on this combination of therapies.

The efficacy of fenofibrate in a fully adherent group was estimated, using randomization-based efficacy estimators or instrumental variable analysis, $[1,10]$ by adjusting for the nonuse of fenofibrate by the following approximation method:

$$
1-H R_{a d j}=(1-H R) /\left(1-D_{F}\right) \lambda_{\mathrm{i}}(t)=\lambda_{0}(t) e^{\mathrm{Xi} \beta+\mathrm{Zi}(\mathrm{t}) \omega}
$$

where $H R_{a d j}$ is the adjusted $\mathrm{HR}$ estimate, $H R$ the unadjusted estimated $\mathrm{HR}$, and $D_{F}$ the proportion of patients discontinuing fenofibrate therapy averaged over the study period [28,29]. An alternative version of this adjustment was undertaken in which $D_{F}$ was the average proportion discontinuing fenofibrate among patients having an event [1]. To avoid potential bias due to treatment decisions that might have been related to the event itself, we excluded from these calculations data from patients starting cardiovascular drugs within 1 month of the event.

All results were unadjusted for multiple comparisons. All analyses used SAS (version 9.1; SAS Institute, Inc. Cary, NC).

\section{Results}

\section{Patient Characteristics and Use of Lipid-Modifying Therapies}

Patient characteristics are shown in Table 1. Lipid-lowering therapy was commenced more often in the group assigned placebo than the group assigned fenofibrate (average use $17 \%$ vs $8 \%$; $P<0.001)$ and more often among the groups with prior cardiovascular disease, dyslipidemia or higher baseline LDL cholesterol levels (each $P<0.001$ ). The most common lipidlowering therapy used was a statin (in $93 \%$ of patients), followed by a fibrate $(6 \%)$, and other $(2 \%)$.
The discontinuation rate of study medication was similar in both randomized groups, steadily increased over time, and averaged $10 \%$ over the follow-up period of 5 years (Table 1 and Figure 1). The rate of discontinuation was somewhat higher than average among patients with prior cardiovascular disease or older age, but was similar for other major groupings. The discontinuation rate among those subsequently having a cardiovascular event was $8.7 \%$ in those assigned to placebo and $13.9 \%$ in those assigned to fenofibrate.

\section{Risk Factors for the Use of Lipid-Modifying Therapies}

In a multivariable analysis of baseline risk factors in the placebo group, higher LDL cholesterol, lower high-density lipoprotein cholesterol, higher systolic blood pressure, lack of obesity, a previous CHD event, and country (Australia or New Zealand) each independently predicted a higher rate of commencing lipidlowering therapy, particularly statins (Table 2). When classified according to a risk score for commencing lipid-lowering therapy, those patients with the highest scores had a significantly higher event rate than those with the lowest scores, indicating that patients at higher risk of cardiovascular disease events were much more likely to start statin therapy (Table S2).

\section{Use of Statin Therapy and Evidence of Reduction in Cardiovascular Disease Events}

Among patients starting statin therapy, simvastatin and atorvastatin were most used (Table 3). On the basis of the metaanalysis by Law et al. [24] and the average daily dosage of statin, the percentage reduction in LDL cholesterol (average for both groups) was estimated as $44 \%, 33 \%$, and $27 \%$ for those on atorvastatin, simvastatin and pravastatin, respectively. Among those who started statin therapy, the average LDL cholesterol before starting therapy was $3.31 \mathrm{mmol} / \mathrm{L}$ in the placebo group and $3.04 \mathrm{mmol} / \mathrm{L}$ in the fenofibrate group; the average absolute reductions in LDL cholesterol were estimated at 1.18 and $1.09 \mathrm{mmol} / \mathrm{L}$, respectively.

The assumed effects of statin therapy on subsequent cardiovascular events were estimated as 27\% and 25\% reductions in CHD events and 25\% and 23\% reductions in cardiovascular events in 
Table 2. Risk factors for starting lipid-lowering therapy during the FIELD study.*

\begin{tabular}{|c|c|c|c|c|}
\hline Risk factor & $\%$ of $\mathbf{4 9 0 0}$ patients* & $\%$ using lipid-lowering therapy $\dagger$ & Adjusted HR $(95 \% \mathrm{Cl})$ & $P$ \\
\hline Country & & & & $<0.001$ \\
\hline Finland & 14 & 18 & 1.00 & \\
\hline Australia & 62 & 36 & $2.03(1.68-2.44)$ & \\
\hline New Zealand & 24 & 48 & $2.25(1.85-2.75)$ & \\
\hline \multicolumn{5}{|l|}{ Clinical history } \\
\hline Prior myocardial infarction & 5.2 & 51 & $1.50(1.23-1.83)$ & $<0.001$ \\
\hline Prior angina & 12 & 47 & $1.43(1.24-1.66)$ & $<0.001$ \\
\hline Prior PTCA & 1.3 & 57 & $1.58(1.12-2.23)$ & 0.01 \\
\hline $\mathrm{BMI} \geq 30 \mathrm{~kg} / \mathrm{m}^{2}$ & 48 & 35 & $0.91(0.83-1.00)$ & 0.04 \\
\hline Systolic blood pressure (mm Hg) & & & & 0.003 \\
\hline$\leq 130$ & 27 & 35 & 1.00 & \\
\hline$>130-140$ & 25 & 36 & $1.14(1.00-1.30)$ & \\
\hline$>140-150$ & 24 & 38 & $1.22(1.07-1.39)$ & \\
\hline$>150$ & 24 & 37 & $1.27(1.11-1.45)$ & \\
\hline LDL cholesterol (mmol/L) & & & & $<0.001$ \\
\hline$<2.52$ & 20 & 18 & 1.00 & \\
\hline $2.52-<2.91$ & 20 & 28 & $1.62(1.34-1.96)$ & \\
\hline $2.91-<3.25$ & 20 & 36 & $2.21(1.84-2.65)$ & \\
\hline $3.25-<3.63$ & 20 & 42 & $2.63(2.20-3.14)$ & \\
\hline$\geq 3.63$ & 20 & 57 & $4.23(3.56-5.03)$ & \\
\hline HDL cholesterol (mmol/L) & & & & $<0.001$ \\
\hline$<0.88$ & 20 & 39 & 1.00 & \\
\hline $0.88-<1.005$ & 20 & 40 & $0.95(0.83-1.10)$ & \\
\hline $1.005-<1.125$ & 20 & 34 & $0.73(0.63-0.84)$ & \\
\hline $1.125-<1.285$ & 20 & 35 & $0.75(0.65-0.87)$ & \\
\hline$\geq 1.285$ & 20 & 34 & $0.78(0.67-0.91)$ & \\
\hline
\end{tabular}

${ }^{*}$ Model derived by using the placebo group only.

Patients who had started using statins, fibrates, resins or other lipid-lowering medications during the trial and had remained on them for at least 3 months in total. "The initial variables were: sex, age, country, clinical history (myocardial infarction, stroke, angina, CABG, PTCA), smoking status, BMI, waist-hip ratio, systolic blood pressure, diastolic blood pressure, LDL cholesterol, HDL cholesterol, triglyceride.

FIELD = Fenofibrate Intervention and Event Lowering in Diabetes; $\mathrm{HR}=$ hazard ratio; $\mathrm{Cl}=$ confidence interval; PTCA = percutaneous transluminal coronary angioplasty; $\mathrm{BMI}=$ body mass index; $\mathrm{HDL}=$ high-density lipoprotein; $\mathrm{LDL}=$ low-density lipoprotein.

doi:10.1371/journal.pone.0008580.t002

the placebo and fenofibrate groups, respectively (Table 4). Very similar estimates were obtained when we applied the results from the GTTC overview of statin therapy for patients with diabetes [23] in a sensitivity analysis.

\section{Use of Other Cardiovascular Medicines and Evidence from Randomized Trials of Cardiovascular Event Reduction}

Use of other cardiovascular drugs was well balanced between treatment arms at baseline (Table 5). These treatments increased over the course of the trial. By the close of the study, antiplatelet therapy was used by half the patients. Use of ACE inhibitors, beta-blockers and diuretics was slightly more common among patients assigned placebo than those assigned fenofibrate (each $P<0.05)$.

\section{Effect of Fenofibrate on Cardiovascular Disease Events}

Before any adjustment for other drugs, fenofibrate was associated with a significant $11 \%$ relative risk reduction in total cardiovascular disease events $(P=0.04)$ and a nonsignificant
$11 \%$ reduction in the primary outcome, CHD events $(P=0.2)$ (Table 6, Figure 2). After adjustment for the effect of statin therapy and other medicines on subsequent cardiovascular disease events, the effect of fenofibrate was moderately larger (Table 6). After the additional adjustment for discontinuation of fenofibrate therapy, efficacy estimates moderately improved to a $15 \%$ reduction in cardiovascular events $(P=0.008)$ and a $16 \%$ reduction in CHD events $(P=0.06)$. About two-thirds of the change in effect due to adjustment for other medicines can be explained by statin use alone (Figure 2). After adjustment for the use of other medicines and discontinuation of fenofibrate, the effect of fenofibrate on nonfatal myocardial infarction increased from $24 \%$ to $30 \%$, on stroke from $10 \%$ to $14 \%$, and on revascularization from $20 \%$ to $25 \%$. The previously reported nonsignificant increase in cardiovascular disease deaths was reduced from $11 \%$ to $8 \%$.

In a sensitivity analysis using the approach suggested by White [1] (using the discontinuation rate only among those having an event), the fully adjusted estimates of the effect of fenofibrate were a $16 \%$ reduction in cardiovascular events and a $17 \%$ reduction in CHD events. 
Table 3. Average use of statins in FIELD and assumed effects on subsequent LDL cholesterol.

\begin{tabular}{|c|c|c|c|c|c|}
\hline Drug & Treatment group & $\begin{array}{l}\text { \% started } \\
\text { statin }\end{array}$ & $\begin{array}{l}\text { Average dose } \\
\text { (mg/day) }\end{array}$ & $\begin{array}{l}\% \text { reduction in } \\
\text { LDL cholesterol* }\end{array}$ & $\begin{array}{l}\text { Assumed change in LDL } \\
\text { cholesterol }(\mathrm{mmol} / \mathrm{L}) \dagger\end{array}$ \\
\hline \multirow[t]{2}{*}{ Atorvastatin } & Placebo & 6.1 & 20.5 & 43 & -1.43 \\
\hline & Fenofibrate & 2.8 & 21.2 & 44 & -1.33 \\
\hline \multirow[t]{2}{*}{ Simvastatin } & Placebo & 8.1 & 24.2 & 33 & -1.09 \\
\hline & Fenofibrate & 3.7 & 25.5 & 33 & -1.01 \\
\hline \multirow[t]{2}{*}{ Pravastatin } & Placebo & 2.4 & 28.9 & 26 & -0.87 \\
\hline & Fenofibrate & 1.4 & 30.0 & 27 & -0.81 \\
\hline \multirow[t]{2}{*}{ Other statin } & Placebo & 0.5 & - & 33 & -1.09 \\
\hline & Fenofibrate & 0.2 & - & 33 & -1.00 \\
\hline \multirow[t]{2}{*}{ Any statin } & Placebo & $16.1 \ddagger$ & - & - & $-1.18 \S$ \\
\hline & Fenofibrate & 7.9 & - & - & $-1.09 \S$ \\
\hline
\end{tabular}

*Derived from meta-analysis of short-term randomized trials of statins [24].

${ }^{\dagger}$ Calculated from the percentage reduction in LDL cholesterol applied to the average prior LDL cholesterol level in each treatment group for those patients who subsequently started lipid-lowering therapy.

\$93\% of patients who started other lipid-lowering treatment took statins.

${ }^{\S}$ Based on a weighted average of LDL change for individual statins.

FIELD = Fenofibrate Intervention and Event Lowering in Diabetes; $L D L=$ low-density lipoprotein.

doi:10.1371/journal.pone.0008580.t003

\section{Treatment Effects Within Subgroups}

Treatment effects within major subgroups are shown in Figure 3 and Table S2. As previously reported, the treatment effect of fenofibrate was apparently larger among patients without prior cardiovascular disease than those with, and among patients aged under 65 years than those aged at least 65 years, as demonstrated by the interaction $P$ values. Such tests for heterogeneity were nominally statistically significant, but only when not adjusted for the multiple subgroup comparisons. The apparent heterogeneity remained similar after adjustment for the differential use of statins and other cardiovascular medicines, but these differences became less significant when other baseline covariates were also adjusted for. Within each subgroup, the HRs became somewhat lower, reflecting the greater effect of fenofibrate after adjustment. There was no consistent pattern of a fenofibrate effect on cardiovascular events by quintile of risk of statin use, either before or after adjustment for use of other medicines (Table S2).

\section{Discussion}

Our method for adjusting a trial result for other active treatments is novel. It extends established methods by adjusting for use of nonstudy medication in a fashion that is not subject to selection bias. As such, this can be thought of as an adjusted intention-to-treat analysis, which better determines the underlying true effect of the study treatment and also estimates an effect for a fully adherent patient group, while avoiding the biases inherent in a per-protocol analysis. In the example of the FIELD study, the likely true effects of treatment were underestimated by more than one-third in the original simple intention-to-treat analysis.

Conventional methods to adjust for both nonadherence and nonstudy treatments after randomization have been subject to selection bias $[1,4,5]$ and higher false-positive rates. Apparent differences in outcomes may be driven by selection of patients for inclusion in analysis, rather than by treatment effects.

Methods that adjust the intention-to-treat analyses according to rates of noncompliance and other factors - randomized-based efficacy estimation methods - avoid selection bias by basing the analyses on the original groups $[1,3,8-11,28-32]$. Approaches to date have largely been restricted to adjustment for the use or nonuse of the trial medicines. In particular, instrumental variable analysis [1,9-11] can estimate average treatment efficacy among compliers, or among all patients under the assumption of full adherence to randomized treatment. Estimates are valid under such an assumption, but, unlike those from per-protocol analyses, do not account for drop-outs being sicker or healthier than those continuing on medication, and these methods do not address the uptake on nonstudy medicines. We adopted a simplified form of this approach for adjusting for the discontinuation of fenofibrate.

Our method of adjustment for use of nonstudy medicines applying external randomized trial evidence for these drugs — uses a randomization-based efficacy estimate that is not subject to selection bias. The method applies the same relative risk reduction (as estimated in other randomized trials) to all patients after they

Table 4. Assumed effects of using statins (\% relative risk reduction*) on subsequent cardiovascular (CVD) events in the FIELD study.

\begin{tabular}{llll}
\hline & Placebo & $\begin{array}{l}\text { Fenofibrate } \\
\text { group } \dagger\end{array}$ & All patients \\
\hline Type of CVD event & 27 & 25 & 26 \\
\hline CHD event & 22 & 21 & 22 \\
CHD death & 31 & 28 & 30 \\
Nonfatal MI & 20 & 18 & 19 \\
CVD death & 20 & 18 & 19 \\
Stroke & 28 & 26 & 27 \\
Revascularization & 25 & 23 & 24 \\
Any CVD event & & &
\end{tabular}

"Estimates of event reduction per $\mathrm{mmol} / \mathrm{L}$ change in $\mathrm{LDL}$ cholesterol were derived from the Cholesterol Treatment Trialists' overview of statin therapy [22].

Assumed absolute change in LDL cholesterol from statin use: $-1.18 \mathrm{mmol} / \mathrm{L}$ in the placebo group and $-1.09 \mathrm{mmol} / \mathrm{L}$ in the fenofibrate group. FIELD = Fenofibrate Intervention and Event Lowering in Diabetes; $\mathrm{CHD}=$ coronary heart disease; $\mathrm{Ml}=$ myocardial infarction.

doi:10.1371/journal.pone.0008580.t004 
Table 5. Percentages of patients using other cardiovascular drugs at baseline and study close, by randomized group, in the FIELD study.

\begin{tabular}{|c|c|c|c|c|}
\hline & Baseline & Baseline & Study close & Study close \\
\hline Type of drug & Placebo $(n=4900)$ & Fenofibrate $(n=4895)$ & Placebo $(n=4900)$ & Fenofibrate $(n=4895)$ \\
\hline Any antiplatelet & 29 & 29 & 51 & 50 \\
\hline Aspirin & 29 & 29 & 47 & 46 \\
\hline Other antiplatelet & 0.6 & 0.3 & 4 & 4 \\
\hline Angiotensin-converting enzyme inhibitor & 34 & 33 & 48 & 45 \\
\hline Angiotensin II receptor antagonist & 5 & 5 & 20 & 20 \\
\hline Beta-blocker & 14 & 15 & 26 & 24 \\
\hline Calcium antagonist & 19 & 20 & 27 & 26 \\
\hline Nitrate & 6 & 5 & 12 & 11 \\
\hline Diuretic & 15 & 15 & 24 & 21 \\
\hline
\end{tabular}

FIELD = Fenofibrate Intervention and Event Lowering in Diabetes

doi:10.1371/journal.pone.0008580.t005

commence such therapies. This assumes that the same relative risk reduction from these treatments would apply to a broad crosssection of patients. This appears to be the case in our example on the basis of evidence from several randomized trials showing no significant heterogeneity of treatment effect across a wide range of subgroups [14,21-23].

There are several examples of placebo-controlled trials in which the uptake of other active treatments differed by randomized group, and where this may have contributed to an underestimation of treatment effect or failure to detect a significant effect for some outcomes. These include trials of antihypertensive therapy or antiplatelet therapies to prevent vascular events. In trials such as Reduction of Endpoints in NIDDM with the Angiotensin II Antagonist Losartan (RENAAL) [33], the Study on Cognition and Prognosis in the Elderly (SCOPE) [34], the Jikei Heart Study [35], and Action in Diabetes and Vascular Disease (ADVANCE) [36], the use of other antihypertensive agents was greater in the placebo group, leading to a likely underestimation of the underlying effect of the trial treatment, and the possibility of missing effects on some outcomes. In the Clopidogrel in Unstable Angina to Prevent Recurrent Events (CURE) trial [37], which evaluated clopidogrel in acute coronary syndromes, use of thrombolytic agents and glycoprotein IIb/IIIa inhibitors was slightly greater in the placebo group. The effects of nontrial treatments on estimates may have been small in some cases, but their effects can be more directly assessed by our approach.

While we support the use of unadjusted intention-to-treat analyses as the primary analysis in randomized trials, it should be recognized that this may underestimate the efficacy of treatment as applied in practice. In our example, the primary results of the FIELD trial based on an intention-to-treat analysis of all patients showed smaller effects of fenofibrate on cardiovascular events than expected in the trial design. The trial was well powered to detect a true $27 \%$ reduction in cardiovascular events, corresponding to an approximate $22 \%$ reduction in the intention-to-treat analysis. The observed effects of fenofibrate were substantially smaller than this, probably in part because the average true effect of the drug is more modest, but also because the results of the intention-to-treat analyses were attenuated by about one-third, owing to substantial uptake during the trial of other medicines, particularly statins, together with the discontinuation of fenofibrate by some patients.

The adjusted analyses suggest that plausible treatment effects of fenofibrate in this setting are a $15 \%$ reduction in all cardiovascular disease events, a $16 \%$ reduction in major CHD events (the primary endpoint), and 30\% reduction in nonfatal CHD events, all of which would make the value of such treatment more

Table 6. Treatment effects of fenofibrate unadjusted and adjusted for the use of other CVD drugs in the FIELD study.

\begin{tabular}{|c|c|c|c|c|c|c|c|}
\hline Outcome & $\begin{array}{l}\text { No. } \\
\text { events }\end{array}$ & $\begin{array}{l}\text { Unadjusted RRR } \\
(95 \% \mathrm{Cl})\end{array}$ & $P$ & $\begin{array}{l}\text { RRR adjusted for use of statins } \\
\text { and other CVD drugs }(95 \% \mathrm{Cl})\end{array}$ & $P$ & $\begin{array}{l}\text { RRR additionally adjusted for } \\
\text { fenofibrate discontinuation* } \\
(95 \% \mathrm{Cl})\end{array}$ & $P$ \\
\hline CHD event & 544 & $11(-5$ to 25$)$ & 0.2 & 15 (-1 to 28$)$ & 0.06 & $16(-1$ to 31$)$ & 0.06 \\
\hline CHD death & 203 & $-19(-57$ to 10$)$ & 0.2 & $-14(-51$ to 13$)$ & 0.3 & $-16(-56$ to 15$)$ & 0.3 \\
\hline Nonfatal MI & 365 & 24 (6 to 38 ) & 0.01 & 27 (10 to 41$)$ & 0.003 & 30 (11 to 45$)$ & 0.003 \\
\hline CVD death & 267 & $-11(-41$ to 13$)$ & 0.4 & $-7(-36$ to 16$)$ & 0.6 & $-8(-40$ to 18$)$ & 0.6 \\
\hline Stroke & 333 & 10 ( -12 to 27$)$ & 0.4 & $12(-9$ to 29$)$ & 0.2 & 14 ( -10 to 33$)$ & 0.2 \\
\hline Revascularization & 851 & 20 (8 to 30$)$ & 0.002 & 22 (11 to 32 ) & $<0.001$ & 25 (13 to 36$)$ & $<0.001$ \\
\hline Any CVD event & 1295 & 11 (1 to 20 ) & 0.04 & 14 (4 to 23$)$ & 0.008 & 15 (4 to 25$)$ & 0.008 \\
\hline
\end{tabular}

*Adjusted for uptake of statins and other drugs and for discontinuation of fenofibrate.

FIELD = Fenofibrate Intervention and Event Lowering in Diabetes; $C V D=$ cardiovascular disease; $\mathrm{RRR}=$ relative risk reduction $(\%)$; $\mathrm{Cl}=$ confidence interval; $\mathrm{CHD}=$ coronary heart disease; $\mathrm{Ml}=$ myocardial infarction

doi:10.1371/journal.pone.0008580.t006 


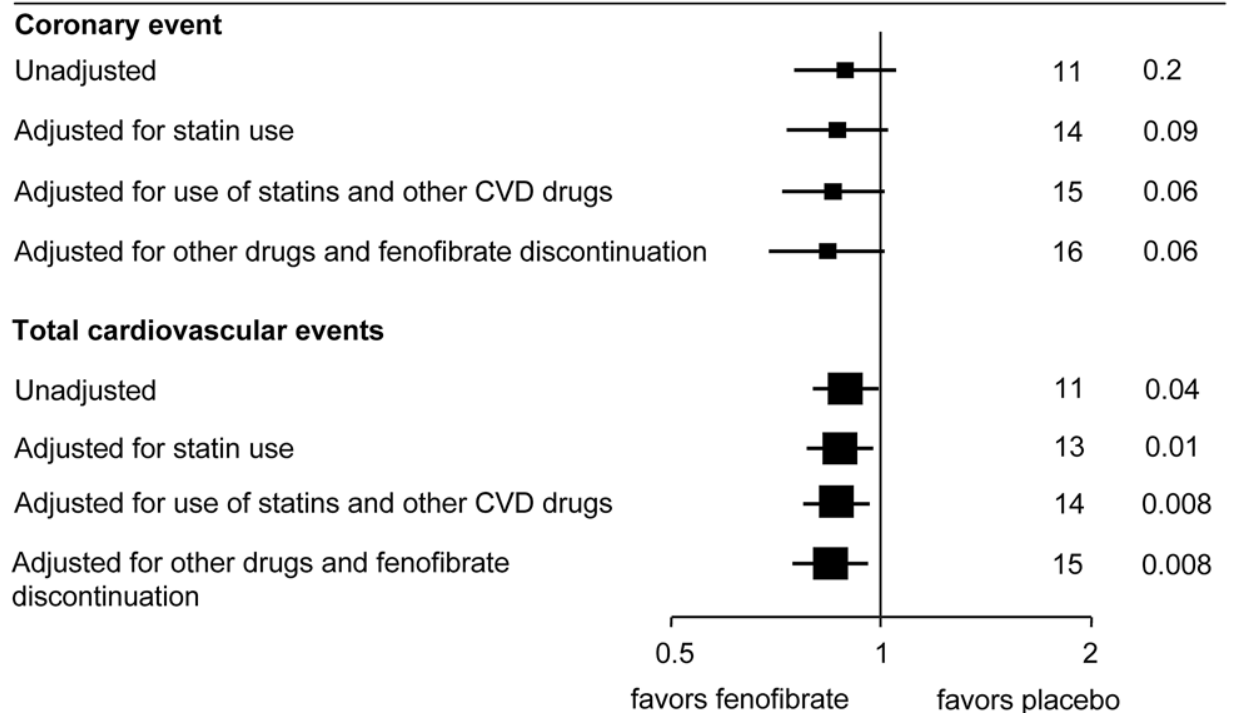

Figure 2. Effects of fenofibrate on events, with and without adjustment for use of statins and other drugs (RRR $=$ relative risk reduction).

doi:10.1371/journal.pone.0008580.g002

compelling. A $15 \%$ reduction would correspond to an absolute reduction in risk of about $2 \%$ or a number needed to treat of 50 patients over 5 years to prevent a major cardiovascular disease event. The adjusted analyses not only indicate a moderately larger treatment effect but also provide strong statistical evidence for a more substantial treatment effect than can be claimed using an intention-to-treat analysis. These analyses also allow some examination of whether the different uptakes of other cardiovascular disease medicines may have been a factor in the apparent variation in treatment effect within subgroups. Statin therapy was more likely to be used by patients with prior cardiovascular disease than not and by patients with more abnormal (than normal) lipid profiles. However, adjustment for the use of statins and other cardiovascular medicines did not appreciably alter the possible heterogeneity for these subgroups, so this does not explain the apparently different treatment effects. As discussed in more detail elsewhere [1], heterogeneity across some subgroups is still consistent with a chance finding and may relate, in part, to some differences in other baseline characteristics.

The methods have some limitations. These include: 1 . the use of the same assumed treatment effect for each drug applied to each individual patient; 2. the use of evidence from different settings than might apply exactly to the trial setting of interest; and 3. the post-hoc nature of the assumptions made in this example, such as the source of external evidence. The post-hoc nature is not a limitation of the method itself, as these concerns could be addressed by building these approaches into the final analysis plan of future trials before unblinding. Also, one could introduce some randomness to the estimates used. In this analysis we chose conservative assumptions or undertook sensitivity analyses of alternative assumptions. For example, we assumed less than a fully multiplicative model when considering multiple drugs in the same patient, and variation in our assumptions resulted in similar conclusions.

Further refinements to the general approach are possible by: 1 . using individual estimates of risk reduction based on a particular drug and dose (rather than applying the average risk reduction to all patients); and 2. considering a random variation in the size of the treatment effect for individual patients. It is recognized that the latter will lead to higher final $P$ values.

Direct validation of this example in another randomized trial will not be possible, as a trial of the same type is no longer possible. However, it will be of interest to see, in the next 6 months, the results of the Action to Control Cardiovascular Risk in Diabetes (ACCORD) trial [38], which is evaluating the additional effect of fenofibrate on a background of all patients receiving statin therapy.

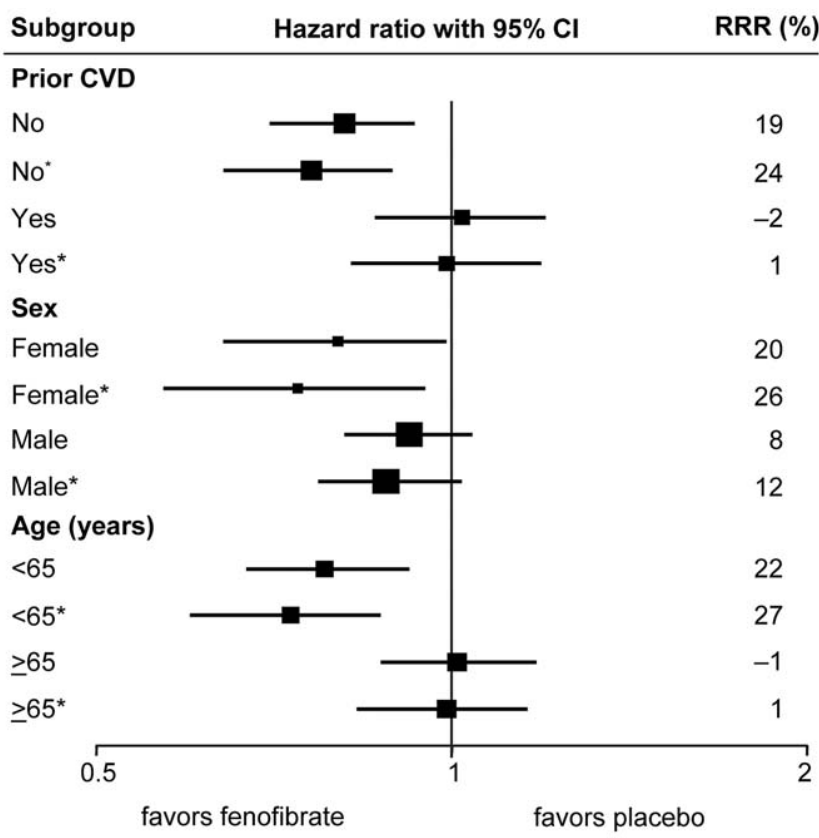

Figure 3. Effects of fenofibrate on cardiovascular events, by major subgroup. *Adjusted for use of other cardiovascular drugs and discontinuation of fenofibrate. $R R R=$ relative risk reduction. doi:10.1371/journal.pone.0008580.g003 
In conclusion, we have adjusted for the effects of an intervention by applying a new method that is not subject to selection bias, that provides an estimate of the underlying treatment effect and that takes into account both adherence to study treatment and the differential use of other nonstudy medicines. The approach suggests a moderate but real underestimate of the effects of fenofibrate on the prespecified cardiovascular outcomes of the FIELD study, which would make a stronger case for using such therapy. The adjusted results should provide more reliable estimates for future clinical decision making. The application of evidence-based estimates related to the use of other nonstudy (cardiovascular) medicines, as described in this setting, may be relevant to many long-term clinical trials, and the approaches adopted here should therefore have wide application and will be especially valuable where differential changes in usual care between treatment arms occur.

\section{Supporting Information}

Table S1 Assumed relative reduction (\%) in major cardiovascular events from other cardiovascular medication.

Found at: doi:10.1371/journal.pone.0008580.s001 (0.03 MB DOC)

\section{References}

1. White IR (2005) Uses and limitations of randomization-based efficacy estimators. Stat Methods Med Res 14: 327.

2. Rosenblum M, Jewell NP, van der Laan M, Shiboski S, Van der Straten A, et al. (2009) Analysing direct effects in randomized trials with secondary interventions: an application to human immunodeficiency virus prevention trials. J R Stat Soc A172: 443-465.

3. Yamaguchi T, Ohashi Y (2004) Adjusting for differential proportions of secondline treatment in cancer clinical trials. Part I: Structural nested models and marginal structural models to test and estimate treatment arm effects. Stat Med 23: 1991-2003.

4. Heritier SR, Gebski VJ, Keech AC (2003) Inclusion of patients in clinical trial analysis: the intention-to-treat principle. Med J Aust 179: 438-440.

5. Sheiner LB, Rubin DB (1995) Intention-to-treat analysis and the goal of clinical trials. Clin Pharmacol Ther 56: 6-10.

6. FIELD Study Investigators (2005) Effects of long-term fenofibrate therapy on cardiovascular events among 9795 people with type 2 diabetes mellitus (the FIELD study): a randomised controlled trial. Lancet 366: 1849-1861.

7. FIELD study investigators (2004) The need for a large-scale trial of fibrate therapy in diabetes: the rationale and design of the Fenofibrate Intervention and Event Lowering in Diabetes (FIELD) study. ISRCTN64783481. Cardiovasc Diabetol 3: 9.

8. Nagelkerke N, Fidler V, Bernsen R, Borgdor M (2000) Estimating treatment effect in randomized clinical trials in the presence of non-compliance. Stat Med 19: $1849-1864$.

9. Little RJ, Long Q Lin X (2009) A comparison of methods for estimating the causal effect of a treatment in randomized clinical trials subject to noncompliance. Biometrics 65: 640-649.

10. Bond SJ, White IR, Walker AS (2007) Instrumental variables and interactions in the causal analysis of a complex clinical trial. Stat Med 26: 1473-1496.

11. Marcus SM, Gibbons RD (2001) Estimating the efficacy of receiving treatment in randomized clinical trials with noncompliance. Health Serv Outcomes Res Methodol 2: 247-258.

12. FIELD Study Investigators (2005) Fenofibrate Intervention and Event Lowering in Diabetes (FIELD) study, a randomized, placebocontrolled trial: baseline characteristics and short-term effects of fenofibrate. Cardiovasc Diabetol 4: 13.

13. Antithrombotic Trialists' Collaboration (2002) Collaborative meta-analysis of randomised trials of antiplatelet therapy for prevention of death, myocardial infarction, and stroke in high risk patients. BMJ 324: 71-86.

14. Wald NJ, Law MR (2003) A strategy to reduce cardiovascular disease by more than 80\%. BMJ 326: 1419-1427.

15. Collins R, Peto R, MacMahon S, Hebert P, Fiebach NH, et al. (1990) Blood pressure, stroke and coronary heart disease. Part 2: short-term reductions in blood pressure: overview of randomised drug trials in their epidemiological context. Lancet 335: 827-838.

16. Yusuf S, Peto R, Lewis J, Collins R, Sleight P (1985) Beta blockade during and after myocardial infarction: overview of randomized trials. Prog Cardiovascular Dis 27: $335-371$.

17. Yusuf S, Wittes J, Friedman L (1988) Overview of results of randomized clinical trials in heart disease. I. Treatments following myocardial infarction. JAMA 260: 2088-2093.
Table S2 Hazard ratios for the effects of fenofibrate on cardiovascular (CVD) events unadjusted and adjusted for the use of statins and other CVD drugs within major subgroups and according to risk of starting CVD drugs.

Found at: doi:10.1371/journal.pone.0008580.s002 (0.06 MB DOC)

\section{Acknowledgments}

We thank Rhana Pike for assistance with the preparation of this manuscript.

\section{Author Contributions}

Conceived and designed the experiments: JJS MV PG JDB RS CP KB AK. Performed the experiments: JJS RO PG JDB RS DRS CE AK. Analyzed the data: JJS MV RO CP KB. Contributed reagents/materials/ analysis tools: JJS JDB RS DRS CE AK. Wrote the paper: JJS MV PG AK. Interpreted analyses and reviewed the manuscript: MV RO PG AK. Reviewed analyses and draft manuscripts and approved final paper: JDB RS. Reviewed and interpreted analyses and draft manuscript: CP DRS CE. Reviewed analyses and draft manuscript: KB.

18. Blood Pressure Lowering Treatment Trialists' Collaboration (2000) Effects of ACE inhibitors, calcium antagonists, and other blood pressure lowering drugs: results of prospectively designed overviews of randomised trials. Lancet 355: 1955-1964.

19. Law MR, Wald NJ, Morris JK, Jordan R (2003) Value of low dose combination treatment with blood pressure lowering drugs: analysis of 354 randomised trials. BMJ 326: 1427-1431.

20. Collins R, MacMahon S (1994) Blood pressure, antihypertensive drug treatment and the risks of stroke and coronary heart disease. Br Med Bull 50: 272-298.

21. Capewell S, O'Flaherty M (2008) Maximising secondary prevention therapies in patients with coronary heart disease. Heart 94: 8-9.

22. Cholesterol Treatment Trialists' (CTT) Collaborators (2005) Efficacy and safety of cholesterol-lowering treatment: prospective meta-analysis of data from 90056 participants in 14 randomised trials of statins. Lancet 366: 1267-1278.

23. Kearney PM, Blackwell L, Collins R, Keech A, Simes J, et al. (2008) Efficacy of cholesterol-lowering therapy in 18,686 people with diabetes in 14 randomised trials of statins: a meta-analysis. Lancet 71: 117-125.

24. Law MR, Wald NJ, Rudnicka A (2003) Quantifying effect of statins on low density lipoprotein cholesterol, ischaemic heart disease, and stroke: systematic review and meta-analysis. BMJ 326: 1423-1427.

25. Lee ET (1992) Statistical Methods for Survival Data Analysis, Second Edition. New York: Wiley.

26. Cox DR (1972) Regression methods of life tables (with discussion). J R Stat Soc B 34: 187-220.

27. Therneau TM, Grambsch PM (2000) Modelling survival data: extending the Cox model. New York: Springer.

28. Newcombe RG (1988) Explanatory and pragmatic estimates of the treatment effect when deviations from allocated treatment occur. Stat Med 7: 1179-1186.

29. Glasziou PP (1992) Meta-analysis adjusting for non-compliance: the example of breast cancer screening. J Clin Epidemiol 45: 1251-1256.

30. Frangakis CE, Rubin DB (1999) Addressing complications of intention-to-treat analysis in the combined presence of all-or-none treatment-noncompliance and subsequent missing outcomes. Biometrika 86: 365-379.

31. Robins JM, Greenland S (1994) Adjusting for differential rates of prophylaxis therapy for PCP in high- versus low-dose AZT treatment arms in an AIDS randomized trial. J Am Stat Assoc 89: 737-749.

32. Cuzick JK, Edwards R, Segnan N (1997) Adjusting for non-compliance and contamination in randomized clinical trials. Stat Med 16: 1017-1029.

33. Brenner BM, Cooper ME, de Zeeuw D, Keane WF, Mitch WE, et al. (2001) Effects of losartan on renal and cardiovascular outcomes in patients with type 2 diabetes and nephropathy. N Engl J Med 345: 861-869.

34. Lithell H, Hansson L, Skoog I, Elmfeldt D, Hofman A, et al. (2004) The Study on Cognition and Prognosis in the Elderly (SCOPE); outcomes in patients not receiving add-on therapy after randomization. J Hypertens 22: 1605-1612.

35. Mochizuki S, Dahlöf B, Shimizu M, Ikewaki K, Yoshikawa M, et al. (2007) Valsartan in a Japanese population with hypertension and other cardiovascular disease (Jikei Heart Study): a randomised, open-label, blinded endpoint morbidity-mortality study. Lancet 369: 1431-149.

36. ADVANCE Collaborative Group (2007) Effects of a fixed combination of perindopril and indapamide on macrovascular and microvascular outcomes in 
Improved Efficacy Estimates

patients with type 2 diabetes mellitus (the ADVANCE trial): a randomised controlled trial. Lancet 370: 829-840.

37. Clopidogrel in Unstable Angina to Prevent Recurrent Events Trial Investigators (2001) Effects of clopidogrel in addition to aspirin in patients with acute coronary syndromes without ST-segment elevation. N Engl J Med 345: 494-502.
38. ACCORD Study Group (2007) Action to Control Cardiovascular Risk in Diabetes (ACCORD) trial: design and methods. Am J Cardiol 99(S): 21i-33i. 